\title{
Situação do controle da transmissão vetorial da doença de Chagas nas Américas
}

\author{
Current situation with Chagas disease vector \\ control in the Americas
}

\footnotetext{
1 Organização Pan-americana da Saúde (OPAS/OMS/Brasil). Setor de Embaixadas Norte, lote 19, sala 19, Brasília, DF 70800-400, Brasil.
}

\begin{abstract}
This article identifies and describes various epidemiological aspects in the natural transmission of Chagas disease in the Americas. It also examines the relative importance of the principal vector species in the disease's transmission and the control levels that are feasible in each instance. Estimations of the population at risk, number of infected cases, and number of chronic cases are presented. Prospects for control are discussed on the basis of past results to predict the expected results with introduced species like Triatoma infestans in the Southern Cone and Rhodnius prolixus in Central America and with the other autochthonous species in areas where they are found. Finally, the article discusses the role of other transmission mechanisms in the maintenance of endemic Chagas disease.
\end{abstract}

Key words Chagas Disease; Triatominae; Insect Vectors; Vector Control; Prevention and Control

Resumo Este trabalho identifica e descreve distintas situações do ponto de vista epidemiológico em relação à transmissão natural da doença de Chagas no continente. A importância relativa das principais espécies de vetor na veiculação da doença e o nível de controle que se pode pretender em cada caso são examinados. Apresentam-se as estimativas existentes no que concerne à população sob risco, bem como ao número de casos de infecção e de doença crônica. Por fim discutem-se as perspectivas do controle, com base nos resultados obtidos e que podem ser colhidos com a introdução de espécies - como Triatoma infestans, no Cone Sul, e Rhodnius prolixus, em parte da América Central - e com as demais espécies, autóctones das áreas onde são encontradas. Além disso, questiona-se o papel que os demais mecanismos de transmissão poderão representar na manutenção da endemia chagásica.

Palavras-chave Doença de Chagas; Triatominae; Insetos Vetores; Controle de Vetores; Prevenção e Controle 


\section{Introdução}

A distribuição de triatomíneos, vetores da doença de Chagas, não se limita ao continente americano, ainda que, em ambiente domiciliar, apenas haja transmissão natural da enfermidade ao homem na região compreendida desde o sul dos Estados Unidos - onde foram reportados apenas poucos casos - até a província de Chubut, na Argentina (Marsden, 1983; WHO, 1991). Disso decorre a designação de Tripanossomíase Americana para a entidade mórbida descrita por Carlos Chagas.

Mesmo nas Américas, a área de dispersão de vetores no ambiente silvestre é bem mais extensa do que aquela com transmissão vetorial. São encontrados triatomíneos entre os $40^{\circ}$ de latitude norte (Zeledón, 1972) e 45o de latitude sul (Jörg, 1957; Bonet, 1972).

Evidentemente, a veiculação da doença no ambiente domiciliar resulta de outras variáveis. É indispensável que o vetor esteja presente, infectado e colonizando a habitação humana. Tal situação, por sua vez, está condicionada primariamente à existência de circunstâncias favoráveis à domiciliação dos vetores, que são condições relacionadas ao ambiente, à ação do homem sobre o ambiente e a atributos do próprio vetor.

A doença de Chagas ocorre caracteristicamente em espaços abertos, naturais ou como produto de ação antrópica. A domiciliação do vetor está relacionada a maior ou menor preservação de seus ecótopos naturais, do tipo de habitação na área e, então, da possibilidade de abrigo para os triatomíneos, do hematofagismo estrito deles e da oferta alimentar existente, bem como do diferente grau de antropofilia de cada uma das espécies (Forattini, 1980).

O ambiente onde se dá a transmissão domiciliar da doença de Chagas é aquele em que as populações sob risco sobrevivem em estado o mais precário e em casa mal construída, acabada ou conservada, expressão da baixa condição econômica e social desses estratos populacionais. Assim, o mapa da distribuição da doença de Chagas coincide, quase sempre, com o da pobreza.

Estima-se que os portadores de infecção chagásica no continente americano sejam entre 18 a 24,7 milhões (Hayes \& Schofield, 1990), ao passo que a população exposta ao risco seja de 90 milhões (UNDP/World Bank/WHO, 1990). Há grande massa de doentes crônicos, o que corresponde a casos produzidos ao longo de muitos anos em que as ações de controle foram apenas pontuais, quando existiram. Apesar da grande magnitude, da transcendência e da com- provada vulnerabilidade ao controle, a prioridade conferida à doença de Chagas foi sempre menor ou apenas precária e instável. $\mathrm{O}$ fato de a doença apresentar caráter crônico e ser predominantemente rural - afetando populações quase sempre à margem do processo produtivo e sem qualquer canal de reivindicação quanto ao atendimento de suas necessidades - é determinante para que a decisão política pelo controle seja - ou, em certos casos, tenha sido dificilmente lograda (Silveira, 1994).

Apesar disso, estudos de custo-benefício ou de custo-efetividade têm mostrado o quanto é compensador o controle relativamente aos gastos com a atenção ao paciente chagásico (Schofield \& Dias, 1991). Análise recente do programa brasileiro de controle da doença de Chagas chegou à estimativa de que, entre 1975 e 1995, teriam sido prevenidas 2.339 .000 novas infecções e 337.000 óbitos ou 11.486.000 Anos de Vida Ajustados para Incapacidade (AVAI). Esses números indicam gastos prevenidos de cerca de US $\$ 17,00$ para cada US $\$ 1,00$ aplicado nas atividades de controle. Semelhante relação coloca as ações de controle da doença de Chagas na categoria das intervenções com alta efetividade (Dariush, 1998).

Em função do baixo nível de prioridade política que mereceu o controle da doença de Chagas, a situação epidemiológica foi sendo agravada em muitos países, mesmo quando já era disponível tecnologia suficiente para o controle da transmissão domiciliar pelo vetor.

\section{A doença}

A prevalência da infecção chagásica nas Américas é conhecida, com maior precisão, no cone sul e Venezuela por intermédio de inquéritos sorológicos mais extensos, os quais serviram para demonstrar a importância da doença como problema de saúde pública, tendo sido determinantes para a instituição ou implementação das ações de controle. Em virtude de ser doença de evolução crônica - dificilmente diagnosticada na fase aguda inicial, porque conta com pouca expressão clínica - exigiu que estudos mais extensos de prevalência fossem promovidos como condição para a decisão de fazer o controle.

Para todos os demais países, as informações são parciais, produto de inquéritos de pequeno alcance e do conhecimento de casos, quase sempre já na fase crônica, ou mediante os serviços de hemoterapia e de fontes como a hemoscopia para o diagnóstico de outras enfermidades. Com base nessas informações frag- 
mentadas é que as estimativas do número de casos existentes de infecção vêm sendo realizadas (Ponce \& Zeledón, 1973; Velazco et al., 1992; Schmunis, 1994; Guhl, 1997).

De acordo com o Banco Mundial, o peso relativo da doença de Chagas - consideradas outras enfermidades transmissíveis endêmicas na América Latina e Caribe e medido por "Anos de Vida Ajustados à Incapacidade" (AVAI) - só é superado pelo conjunto das enfermidades diarréicas, infecções respiratórias e AIDS, correspondendo a mais do que o dobro da soma de malária, esquistossomose, leishmanioses e hanseníase.

Assumindo que $10 \%$ das infecções evoluiriam para formas crônicas com graus variáveis de comprometimento, estima-se que sejam de aproximadamente 1,8 a 2,5 milhões os portadores de doença clínica, com cardiopatia e/ou manifestações digestivas, quase sempre megaesôfago ou megacólon. Tomando-se em conta que esses casos demandam algum tipo de assistência médica - com freqüência, especializada e cirúrgica -, pode-se inferir os custos resultantes.

É preciso mencionar que a reiteração com que a infecção se manifesta clinicamente é variável, consideradas diferentes áreas geográficas. Há evidências a respeito da desproporção entre os casos de infecção e doença no sul do Brasil e Uruguai - o que ganha consistência pelo relativamente pequeno número de óbitos atribuídos à doença de Chagas - ou em países da América Central, como algumas vezes referido para a Costa Rica.

Por sua vez, tudo indica haver diferente tropismo de distintas cepas de Trypanosoma cru$z i$, o que explicaria, por exemplo, o fato de ser mais comum o diagnóstico de formas digestivas na região central do Brasil ou de ser baixa a prevalência de cardiopatias no norte do Equador em relação à região sul (WHO, 1997).

A crença de que a doença teria caráter "benigno" em determinadas áreas pode decorrer do desconhecimento ou da falta de melhor investigação. Em nenhuma hipótese, tal argumento pode servir para que não se faça o controle, uma vez que se tenha conhecimento do risco de transmissão.

\section{Vetores}

São muitos os vetores potenciais da doença de Chagas, mas a maior parte das espécies de triatomíneos conhecidas não tem participação direta na transmissão domiciliar da doença.

Aqui admite-se a existência de 112 espécies no continente americano, bem como a possibilidade de que mudanças em alguns conceitos taxonômicos possa alterar esse quadro, sempre sujeito a modificações pela permanente atualização das informações (Lent \& Wigodzinsky, 1979; Carcavallo et al., 1997).

Sob o ponto de vista epidemiológico, várias categorizações têm sido feitas no sentido de melhor orientar as operações de controle, uma vez que servem à determinação da maior ou menor importância relativa de cada uma das espécies na transmissão da doença ao homem.

Assim, interessa o agrupamento em função da maior ou menor adaptação ao domicílio, classificação mais comumente empregada (Zeledón, 1974; WHO, 1991; Dias, 1992), que aqui se procurou atualizar a partir de acontecimentos mais ou menos recentes (Tabela 1), como a detecção de colônias intradomiciliares de Triatoma rubrovaria e Rhodnius neglectus em áreas originalmente infestadas por Triatoma infestans (Diotaiuti \& Silveira, 1983; Silveira \& Rezende, 1994), ou o achado de exemplares - mesmo que raros e que fossem exclusivamente adultos - de Psamolestes coreodes e de Microtriatoma trinidadensis no ambiente domiciliar (Silveira et al., 1984), ou ainda o processo incipiente de colonização em ecótopos artificiais de Panstrongylus lutzi (Silveira, 1984) e Panstrongylus geniculatus (Valente, 1995).

O certo é que mudanças ambientais, inclusive aquelas decorrentes das próprias ações de controle, podem implicar o reposicionamento de determinada espécie. Com isso, qualquer classificação que se venha a fazer, estará sempre sujeita a revisão.

Além da adaptação ao domicílio, é evidente que a capacidade vetorial depende de outras variáveis como, por exemplo, antropofilia, infectividade ou o tempo entre repasto e dejeção para diferentes espécies (Silva et al., 1993). Em função disso, classificações diferentes têm sido adotadas na definição de prioridades para efeito de intervenção (MS, 1994; Silveira, 1997).

Uma categorização possível e que importa para o controle - em especial, para que se definam claramente os objetivos ou o nível de controle que se pode esperar - considera a área de origem da espécie presente (Silveira, 1985). Nesse caso, duas são as situações possíveis: espécies nativas e introduzidas. Espécies nativas são aquelas autóctones ou indígenas daquele ecossistema natural e que, como tal, podem reinvadir e recolonizar domicílios submetidos a tratamento químico com inseticidas. O tempo para que isso ocorra é função da densidade das populações do vetor em ecótopos silvestres, da própria densidade desses ecótopos e da relação que o homem tem com eles. Assim sendo, não é possível sua eliminação definitiva 
8) México

Vetores: Triatoma barberi, Triatoma dimidiata e Triatoma phyllosoma.

Em alguns dos países do Caribe e nos Estados Unidos da América há vetores naturalmente infectados, sabendo-se que existe o ciclo enzoótico de transmissão, com a infecção comprovada em animais silvestres. A infecção no homem tem caráter esporádico e acidental.

É evidente que cada uma dessas situações deve merecer tratamento diverso e ajustado ao diferente comportamento dos vetores e de sua maior ou menor transcendência e vulnerabilidade às ações de controle.

\section{Controle dos vetores}

Por limitações do conhecimento e por peculiaridades próprias da epidemiologia da doença de Chagas, o controle de populações domiciliadas do vetor é, até o presente, a única possibilidade de reduzir ou, em alguns casos, interromper a transmissão domiciliar da doença.

Entre as limitações de natureza epidemiológica, a primeira consiste em a doença de Chagas ser primitivamente uma enzootia e, como tal, dever subsistir, havendo sempre o risco de domiciliação de vetores desde o ambiente silvestre, além da possibilidade de infecção humana acidental. Isso determina que a doença não seja erradicável. Outro importante fator limitante é o grande número de reservatórios animais, domésticos e silvestres, o que faz com que não se possa pretender o controle pelo esgotamento das fontes de infecção. Afora isso, como já mencionado, é preciso tomar em conta a pouca ou nenhuma expressão clínica da infecção aguda, quando se sabe o tratamento específico eficaz. Por sua vez, não se conhece vacina, e as drogas disponíveis não podem ser utilizadas em larga escala, em função dos paraefeitos que produzem.

Assim, como medida de proteção específica no período pré-patogênico, resta apenas o controle do vetor, o qual pode ser exercido tanto pelo tratamento químico de habitações infestadas com inseticidas de ação residual quanto pela melhoria das condições de habitação, o que, no caso particular da doença de Chagas, pode ser entendido não apenas como medida de promoção de amplo alcance, mas também como ação precípua de proteção - em particular, quando localizada e ajustada a hábitos de determinado vetor. Exemplos disso seriam a substituição da cobertura de palha em casas infestadas por Rhodnius prolixus ou a construção de pisos em casas com infestação por Triatoma dimidiata (Zeledón, 1981).
A melhoria da habitação, no entanto, em razão do relativamente alto custo e de dificuldades de outra natureza - como a posse da terra tem uso restrito e deve ser reservada a situações particulares. Entre essas, a persistência de focos residuais de infestação em áreas submetidas a sucessivos ciclos de tratamento domiciliar com inseticidas, ou a presença de espécies nativas em alta densidade no ambiente silvestre e com grande capacidade invasiva da habitação humana.

A comprovada eficácia do controle pela borrifação sistemática de casas infestadas recomenda seu emprego extensivo como primeira alternativa a ser observada. Tal eficácia desde há muito foi demonstrada. Em 1947 provou-se, em laboratório, a alta ação tóxica do isômero gama do hexaclorociclohexano para triatomíneos (Busvine \& Barnes, 1947). No ano seguinte, no Brasil e na Argentina, foi confirmada em campo a possibilidade de controle de populações domiciliadas do vetor pelo uso regular daquele "novo" inseticida clorado de ação residual, o "Gamexane P 530" (Dias \& Pellegrino, 1948; Romaña \& Abalos, 1948).

Como conseqüência dos resultados obtidos com essas provas de campo, a partir de 1950 foram promovidas, no Brasil, as primeiras "Campanhas contra a doença de Chagas” pelo então Serviço Nacional de Malária.

Na Argentina, trabalhos de caráter ainda experimental e de pequeno alcance foram iniciados na década de 50, conduzidos por Cecilio Romaña na província do Chaco, por Carlos Soler em La Rioja e por Carlos Bravo em Catamarca. Em 1962, as atividades de controle foram assumidas institucionalmente em caráter prioritário e ganharam dimensão nacional, com a criação do "Servicio Nacional de Chagas".

Outro país com história mais remota de controle é a Venezuela. Apesar de a doença de Chagas ter sido pela primeira vez aí reportada em 1919 por Tejera, as ações de controle foram instituídas apenas em 1966, quando se estruturou formalmente a "Campaña Nacional de la Enfermedad de Chagas", o que deu seqüência à luta de Gabaldón, que vinha fazendo, desde 1948 , a defesa da prioridade que deveria merecer o controle da enfermidade, ou seja, desde quando se demonstrou haver instrumentos eficazes de combate aos triatomíneos.

Esses países foram os precursores do controle da transmissão vetorial - ao menos, em mais larga escala -, mas as ações não obedeciam, de início, aos requisitos de continuidade no tempo e contigüidade espacial, condições necessárias à sustentabilidade ou permanência dos resultados, que foram, desse modo, qua- 
se sempre limitados e provisórios. Semelhante situação deveu-se basicamente ao aporte irregular de recursos, nunca suficientes para dar integral cobertura à área com risco de transmissão vetorial.

No caso da Venezuela, os resultados foram bem mais duradouros em função dos investimentos feitos em melhoria habitacional - ações que antecederam o controle químico mais sistematizado e que não eram inicialmente dirigidas exclusivamente ao controle da doença de Chagas, além de terem tido mais o caráter de promoção das condições de vida das populações rurais (“Programa Nacional de Vivienda Rural", 1958).

Durante as décadas de 60 e 70 foram inauguradas ações regulares de controle em países do Cone Sul e Venezuela, as quais foram implementadas na década de 80 e expandidas nos anos 90 , alcançando outros países. Em alguns casos, isso dependeu da resolução, ao menos parcial, de outros problemas de saúde pública, igualmente de grande magnitude, como a malária. A expansão do controle vetorial da doença de Chagas dependeu também da demonstração de resultados, como aqueles colhidos na Argentina, Brasil, Chile, Uruguai e Venezuela.

A dispersão por Triatoma infestans vem sendo progressivamente focalizada. No Brasil, dentre 711 municípios inicialmente infestados, 105 mantinham-se positivos em 1997, mas com taxas de infestação domiciliar e densidade extremamente baixas, com apenas 491 unidades domiciliares infestadas e 1.080 exemplares capturados. O mesmo observa-se para a Argentina, Chile e Uruguai.

O impacto das ações de controle vetorial vem sendo também avaliado através de inquéritos sorológicos em grupos etários jovens ou em estudos de coorte (WHO, 1997).

$\mathrm{Na}$ Argentina, a sorologia em conscritos para o serviço militar mostrou redução de prevalência da infecção de 4,6 a 1,2 em período que corresponde aos últimos dez anos. Entre crianças de 0 a 4 anos de idade, a soro-reatividade foi de apenas $0,9 \%$. No Brasil, as taxas iniciais determinadas mediante inquérito sorológico nacional, que amostrou a população rural entre 1975 e 1980 - foi de 4,2 \% e tem revelado, até o presente, soropositividade média inferior a $0,2 \%$ em inquéritos entre escolares no grupo etário de 7 a 14 anos de idade, realizados em municípios onde a transmissão estaria interrompida com base em indicadores entomológicos. No Chile, a infecção em menores de 15 anos de idade, em 1986, foi de $9,1 \%$, enquanto em inquérito recente foi de 1,9\% no mesmo grupo de idade. Para o Uruguai, o impacto sobre os níveis de prevalência da infecção foi ain- da mais dramático, de 2 a 6\%, em 1986, na população geral, para zero em 1996, no grupo de idade de 0 a 5 anos. No caso da Venezuela, as ações de controle sobre Rhodnius prolixus determinaram também redução importante na prevalência da infecção humana. Entre 1990 e 1994, com mais de 6.300 reações sorológicas em crianças de 0 a 4 anos, apenas $0,6 \%$ dos soros foram reagentes (Salvatella, 1993; Silveira \& Rezende, 1994; Aché \& Berti, 1995; WHO, 1997).

Esses resultados foram determinantes para que outros países, também organizados regionalmente, passassem a priorizar o controle da enfermidade a partir de 1991, com a iniciativa dos países do Cone Sul para a "Eliminação do Triatoma infestans e controle da transmissão transfusional da doença de Chagas", compromisso formalmente assumido pelos Ministros de Saúde da sub-região. Assim aconteceu com o Grupo Andino e os países da América Central, que se incorporaram ao esforço continental para o enfrentamento do problema, um dos mais sérios no que se refere à saúde e que acomete a população rural latino-americana.

\section{Perspectivas}

É evidente que a solução definitiva da transmissão vetorial domiciliar da doença de Chagas depende da melhoria das condições de vida de grande parte da população do continente, sobretudo no meio rural, mas é igualmente certo que há hoje acumulação técnica suficiente para impedir a produção de novos casos da enfermidade.

A transmissão vetorial no caso de T. infestans e R. prolixus, quando domiciliado, pode ser completamente interrompida pelo tratamento químico das habitações (Dujardin et al., 1994; Schmunis, 1994).

No caso de todos os outros vetores quase completamente controlados, deve-se fazer a aplicação de inseticidas e, a partir disso, impedir a recolonização dos domicílios pela manutenção de vigilância entomológica de caráter permanente, contínua, com a indispensável participação da população e o envolvimento da rede de serviços de saúde (Souza et al., 1984; Paulone et al., 1988; García-Zapata \& Marsden, 1994). O retratamento das casas deverá ser feito apenas quando detectada a presença de colônias no interior dos domicílios ou, em alguns casos - como o de espécies com alto potencial vetorial, em função de sua antropifilia e infectividade -, recomenda-se o tratamento periódico peridomiciliar, no sentido de reduzir a pressão de reinfestação. 
No entanto, para o apuro da vigilância é necessário o desenvolvimento de técnicas simples, de baixo custo e mais sensíveis na detecção do vetor, sobretudo quando a densidade é baixa. Apenas assim poder-se-á viabilizar as ações na ausência da doença e ter oportunidade de êxito na intervenção com inseticidas quando necessário.

A transmissão transfusional tende a ser controlada pelo desenvolvimento de técnicas sempre mais sensíveis e de processamento em série de amostras de sangue na triagem de doadores em serviços de hemoterapia. A cobertura vem sendo progressivamente ampliada.

Em termos relativos a outros mecanismos, a transmissão congênita não representa muito em áreas ainda com a transmissão natural mantida, mas pode vir a ser a única via de manutenção da endemia chagásica com a transmissão vetorial e transfusional controlada. É recomendável tornar rotineira a investigação de gestantes chagásicas no pré-natal e garantir o tratamento de recém-nascidos infectados. As estimativas existentes são de que a transmis- são transplacentária é responsável, no momento, pela ocorrência de 9 a 10 mil novos casos nas Américas a cada ano.

O risco de transmissão, com o controle que se considera perfeitamente possível das vias vetorial domiciliar, sangüínea e materno-infantil, estará reduzida a transplante de órgãos ou a acidentes, entendendo-se a transmissão "per os" também como acidental.

A enzootia chagásica, contudo, seguirá existindo e, com isso, a transmissão ocasional pelo vetor será sempre uma possibilidade mesmo no ambiente natural, como conhecido no caso de contato do homem com focos silvestres, fato que, se estiver relacionado com atividade laboral, como no extrativismo de palmeiras, poderá ter significação epidemiológica (Silveira \& Passos, 1986; Coura et al., 1994). Em vista do exposto, não se pode pretender a completa erradicação da doença de Chagas, mas o controle da transmissão endêmica, por toda a prática de controle e experiência acumulada, é absolutamente factível com a tecnologia hoje disponível.

\section{Referências}

ACHE, A. \& BERTI, J., 1995. Programa de Control de la Enfermedad de Chagas en Venezuela. In: Internacional Workshop on Population Genetics and Control of Triatominae, Proceedings (C. J. Schofield, J. P. Dujardin \& J. Jouberg, eds.), pp. 19-25, Santo Domingo de los Colorados: European Community Latin America Triatomine Research Network ECLAT.

BONET, A. H., 1972. Epidemiología de la enfermedad de Chagas en la República Argentina. In: Simposio Internacional de Enfermedad de Chagas, Resúmenes, p. 163, Buenos Aires: Ministerio de la Salud y Acción Social.

BUSVINE, J. R. \& BARNES, S., 1947. Observations on mortality among insects exposed to dry insecticidal fims. Bulletin of Entomological Research, 38: 80-81.

CARCAVALlO, R. U.; GIRON, I. G.; JURBERG, J. \& LENT, H., 1997. Atlas dos Vetores da Doença de Chagas nas Américas. Rio de Janeiro: Editora Fiocruz.

COURA, J. R.; BARRET, T. V. \& ARBOLEDA-NARANJO, M., 1994. Ataque de populações humanas por triatomíneos silvestres no Amazonas: Uma nova forma de transmissão da infecção chagásica? $R e$ vista da Sociedade Brasileira de Medicina Tropical, 27:251-253.

DARIUSH, A., 1998. Análise de Custo-Efetividade do Programa de Controle da Doença de Chagas no Brasil: Relatório Final. Brasília: Organização Panamericana da Saúde.

DIAS, E. \& PELLEGRINO, J., 1948. Alguns ensaios com o "Gamexanne" no combate aos transmissores da doença de Chagas. Brazil Médico, 62:185-190.
DIAS, J. C. P., 1992. Epidemiology of Chagas' disease. In: Chagas' Disease (American Tripanosomiasis): Its Impact on Transfusion and Clinical Medicine (S. Wendel, Z. Brener, M. E. Camargo \& A. Rassi, eds.), pp. 49-80, São Paulo: International Society of Blood Transfusions.

DIOTAIUTI, L. \& SILVEIRA, A. C., 1983. Domiciliação de Rhodnius neglectus Lent, 1954, no estado de Goiás. In: X Reunião Anual de Pesquisa Básica em Doença de Chagas. Programa e Resumos, p. 58, Caxambu: Conselho Nacional de Desenvolvimento Científico e Tecnológico.

DUJARDIN, J. P.; MUÑOZ, M.; CHAVEZ, T.; PONCE, C.; MORENO, J. \& SCHOFIELD C. J., 1998. The origin of Rhodnius prolixus in Central America. Medical and Veterinary Entomology, 12:113-115.

FORATTINI, O. P., 1980. Biogeografia, origem e distribuição da domiciliação de triatomíneos no Brasil. Revista de Saúde Pública, 14:265-299.

GARCIA-ZAPATA, M. T. \& MARSDEN, P. D., 1994. Enfermedad de Chagas: Control y vigilancia com insecticidas y participación comunitaria en Mambaí. Goiás. Brasil. Boletín de la Oficina Sanitaria Panamericana, 116:97-110.

GUHL, F., 1997. Programas para la Eliminación de la Transmisión de la Enfermedad de Chagas en los Países Andinos. Bogotá: Universidad de los Andes. (mimeo.)

HAYES, R. J. \& SCHOFIELD, C. J., 1990. Estimación de las tasas de incidencia de infecciones y parasitosis crónicas a partir de la prevalencia: La enfermedad de Chagas en América Latina. Boletín de la Oficina Sanitaria Panamericana, 108:308-316. 
JÖRG, M. E., 1957. Límite sur de la dispersión geográfica de Triatoma infestans y su infestación por Trypanosoma cruzi en Argentina. Boletín de la Oficina Sanitaria Panamericana, 42:59.

LENT, H. \& WIGODZINSKY, P., 1979. Revision of Triatominae (Hemiptera: Reduviidae) and their significance as vectors of Chagas' disease. Bulletin of the American Museum of Natural History, 163: 127-520.

MARSDEN, P. D., 1983. The transmission of Trypanosoma cruzi to man and its control. In: Human Ecology and Infectious Diaseases (N. A. Croll \& J. H. Cross, eds.), pp. 253-289, London: Academic Press.

MS (Ministério da Saúde), 1994. Controle da Doença de Chagas - Diretrizes Técnicas. Brasília: Fundação Nacional de Saúde.

PAULONE, I.; CHUIT, R.; PEREZ, A.; WISNIVESKYCOLLI, C. \& SEGURA, E. L., 1988. Field research on a epidemiological surveillance alternative of Chagas' disease transmission: The primary health care (PHC) strategy in rural areas. Revista Argentina de Microbiología, 20:103-105.

PONCE, C. \& ZELEDON, R., 1973. La enfermedad de Chagas en Honduras. Boletín de la Oficina Sanitaria Panamericana, 9:239-248.

ROMAÑA, C. \& ABALLOS, J. W., 1948. Acción del “Gamexanne" sobre los triatomídeos. Control domiciliario. Anales del Instituto de Medicina Regional de Tucumán, 2:95-106.

SALVATELLA, R., 1993. Enfermedad de Chagas: Informe de situación en Uruguay. Revista Médica de Uruguay, 9:65-66.

SCHMUNIS, G. A., 1994. American Trypanosomiasis as a public health problem. In: Chagas'Disease and the Nervous System (Pan American Health Organization, ed.), Publicación Científica 574, pp. 3-29, Washington, D.C.: Pan American Health Organization.

SCHOFIELD, C. J. \& DIAS, J. C. P., 1991. A cost benefit analysis of Chagas' disease control. Memórias do Instituto Oswaldo Cruz, 86:285-295.

SCHOFIELD, C. J. \& DUJARDIN, J. P., 1997. Chagas' disease vector control in Central America. Parasitology Today, 13:141-144.

SEGURA, E. L.; ESQUIVEL, M. L.; SALOMON, O.; GOMEZ, A.; SOSA-ESTANI, S. A.; LUNA, C. A.; TULIAN, L.; HURVITZ, A.; BLANCO, S.; ANDRES, A.; ZARATE, J. \& CHUIT, R., 1994. Participación comunitaria en el programa nacional de control de la enfermedad de Chagas. Medicina (Buenos Aires), 54:610-611.

SILVA, I. G.; LUQUETTI, A. O. \& SILVA, H. H. G., 1993. Importância do método de obtenção das dejeções de triatomíneos na avaliação da suscetibilidade triatomínica para Trypanosoma cruzi. Revista da Sociedade Brasileira de Medicina Tropical, 26:19-24.

SILVEIRA, A. C. \& PASSOS, A. D. C., 1986. Altos índices de prevalência sorológica da infecção chagásica em área da Amazônia. Revista da Sociedade Brasileira de Medicina Tropical, 19 (Sup.):45.

SILVEIRA, A. C. \& REZENDE, D. F., 1994. Epidemiologia e controle da transmissão vetorial da doença de Chagas. Revista da Sociedade Brasileira de Medicina Tropical, 27:11-22.

SILVEIRA, A. C., 1984. Altas taxas de infecção natural em Panstrongylus lutzi Neiva \& Pinto, 1923. In: XI
Reunião Anual de Pesquisa Básica em Doença de Chagas, Programa e Resumos, p. 176, Caxambu: Conselho Nacional de Desenvolvimento Científico e Tecnológico - CNPq.

SILVEIRA, A. C., 1985. Respostas às ações de controle da doença de Chagas para espécies de triatomíneos mais e menos adaptadas ao domicílio. Nativas e introduzidas. In: XXI Congresso da Sociedade Brasileira de Medicina Tropical, Resumos, pp. 37-38, São Paulo: Sociedade Brasileira de Medicina Tropical.

SILVEIRA, A. C., 1994. Transcendência da doença de Chagas e prioridade conferida ao controle. Revista da Sociedade Brasileira de Medicina Tropical, 27:100-101.

SILVEIRA, A. C., 1997. Profilaxia da doença de Chagas. In: Trypanosoma cruzi e Doença de Chagas (Z. Brener \& Z. Andrade, eds.), 2a ed., pp. 75-87, Rio de Janeiro: Editora Guanabara-Koogan.

SILVEIRA, A. C.; FEITOSA, V. R. \& BORGES, R., 1984. Distribuição de triatomíneos capturados no ambiente domiciliar, no período 1975/83, no Brasil. Revista Brasileira de Malariologia e Doenças Tropicais, 36:15-312.

SOUZA, A. G.; VALÉRIO-WANDERLEY, D. M.; BURALLI, G. M. \& ANDRADE, J. C. R., 1984. Consolidation of the control of Chagas' disease in the state of São Paulo. Memórias do Instituto Oswaldo Cruz, 79:125-131.

UNDP/World Bank/WHO (United Nations Development Program/World Bank/World Health Organization), 1990. Special Programme for Research and Training in Tropical Diseases. TDR News, 34:3-34.

VALENTE, V. C., 1995. Sobre a Possibilidade de Domiciliação de Panstrongylus geniculatus (Latreille, 1811) e a Transmissão de Casos Autóctones de Doença de Chagas em Muaná, Região do Marajó, Estado do Pará. Monografia, Rio de Janeiro: Escola Nacional de Saúde Pública, Fundação Oswaldo Cruz.

VELAZCO-CASTREJON, O.; VALDESPINO, J. L.; TAPIACONIER, R. \& SALVATIERRA, B., 1992. Seroepidemiología de la enfermedad de Chagas en México. Salud Pública de México, 34:186-196.

WHO (World Health Organization), 1991. Control of Chagas Disease. Technical Report Series 811. Geneva: WHO.

WHO (World Health Organization), 1997. Prospects for the Elimination of Some TDR Diseases. TDR/Gen/ 97.1. Geneva: WHO.

WHO/PAHO (World Health Organization/Pan American Health Organization), 1997. Manual para el Control de Enfermedades Transmisibles. Publicación Científica 564. Geneva: WHO/PAHO.

ZELEDON, R., 1972. Los vectores de la enfermedad de Chagas en América. In: Simposio Internacional de Enfermedad de Chagas, Resúmenes, p. 327, Buenos Aires: Ministerio de Salud y Acción Social.

ZELEDON, R., 1974. Epidemiology, modes of transmission and reservoir host of Chagas' disease. $\mathrm{Ci}$ ba Foundation Symposium, 20:51-77.

ZELEDON, R., 1981. El Triatoma dimidiata y su Relación com la Enfermedad de Chagas. San José: Editorial Universidad Estatal a Distancia. 